\title{
Géolinguistique
}

$14 \mid 2013$

Varia

\section{L'inventaire des microtoponymes : enjeux théoriques et méthodologiques}

The Inventory of Microtoponyms: Theoretical and Methodological Issues

Jean-Pierre Gerfaud et Noël Poncet

\section{Q OpenEdition}

\section{Journals}

Édition électronique

URL : http://journals.openedition.org/geolinguistique/765

DOI : 10.4000/geolinguistique.765

ISSN : 2650-8176

Éditeur

UGA Éditions/Université Grenoble Alpes

\section{Édition imprimée}

Date de publication : 15 décembre 2013

Pagination : $7-20$

ISBN : 978-2-84310-264-6

ISSN : 0761-9081

Référence électronique

Jean-Pierre Gerfaud et Noël Poncet, "L'inventaire des microtoponymes : enjeux théoriques et méthodologiques », Géolinguistique [En ligne], 14 | 2013, mis en ligne le 30 avril 2019, consulté le 05 novembre 2020. URL : http://journals.openedition.org/geolinguistique/765 ; DOI : https://doi.org/ 10.4000/geolinguistique.765 


\title{
Les noms de lieux. L'inventaire des microtoponymes : enjeux théoriques et méthodologiques
}

\author{
Jean-Pierre Gerfaud et Noël Poncet \\ Institut Pierre Gardette, Université catholique de Lyon
}

\section{Résumé}

La présente contribution porte sur une réflexion autour des questions théoriques et méthodologiques liées à l'étude des toponymes. Désigner des lieux par les mots, c'est donner une réponse et du sens à la fois à ses propres besoins et aux besoins communautaires. Se donner des points de repère dans son environnement proche ou éloigné est une nécessité pour tout être animé, en particulier pour tout être humain. Plus précisément, l'étude en question porte sur la création de noms de lieux sur des espaces ou territoires limités, comme ceux de Mérignat, une commune située dans le département de l'Ain (Rhône-Alpes, France).

\section{Mots-clés}

(Micro)toponymie, géolinguistique, motivation linguistique.

\begin{abstract}
This paper aims to provide elements of reflection on the theoretical and methodological issues in the study of toponyms. Designating places by words means providing an answer and a meaning to both one's own needs and community needs. Giving themselves benchmarks in its immediate or remote environment is a necessity for every animated being, especially for all human beings. More specifically, this study focuses on the creation of place names on limited areas or territories, such as those of Mérignat, a village in the Ain province (Rhône-Alpes, France).
\end{abstract}




\section{Keywords}

(Micro)toponymy, linguistic geography, linguistic motivation.

Se donner des points de repère dans son environnement proche ou éloigné est une nécessité pour tout être animé, en particulier pour tout être humain. Cette nécessité répond individuellement aussi bien à des besoins biologiques qu'à des besoins psychosociologiques et symboliques. Cette nécessité est d'autant plus forte que, faisant partie de diverses communautés humaines, l'être humain se doit de communiquer pour répondre aux divers besoins de celles-ci. À travers ce repérage, l'être humain, qui est capable de désigner des réalités célestes en dénommant les constellations, est capable de donner du sens, qui est à la fois direction et signification. Si désigner des lieux par les mots, c'est donner une réponse et du sens à ses propres besoins, c'est aussi donner une réponse et du sens aux besoins communautaires. Avec le temps, cette nécessité s'est renforcée, s'est progressivement complexifiée, s'est universalisée, au point que la réponse par la dénomination des lieux locaux, devenus lieux-dits, tend à devenir la propriété de tous par l'intermédiaire des moyens modernes de communication. On peut être tenté de réduire les résultats de cette complexification à deux termes que propose la linguistique, dénotation et connotation, mais il conviendra d'enrichir ces concepts par les apports de la réalité, elle-même lue à travers les diverses approches de la connaissance.

On peut donc concrètement se demander ce qui motive et encadre la création de noms de lieux sur des espaces ou territoires limités, ceux d'une commune de 315 hectares, comme Mérignat dans le département de l'Ain, soit une centaine de noms de lieux. Une telle surface correspond à la distance $(5,5 \times 5,5 \mathrm{~km})$ que peut parcourir le vigneron qui exploite sa vigne située de part et d'autre d'un col; ce col est évoqué en 1631 par le voyageur allemand Abraham Golnitz.

\section{Les diverses motivations de la désignation et de la dénomination des lieux de l'environnement}

Tout déplacement dans l'espace, même le plus limité, exige une désignation : si cela est vrai pour la femme du Paléolithique qui rappelle son enfant près d'elle ou à l'abri sous la roche où le groupe se protège, cela est encore plus vrai pour les chasseurs ou les cueilleurs partis à la recherche de nourriture qui expliquent ou renseignent sur l'endroit où ils en ont trouvé : ils 
reviennent de tel endroit; ils sont passés par là; là, ils ont trouvé telles baies ou tel animal; ils invitent leurs congénères à y aller. Ils peuvent même ajouter les proximités : c'est à côté de tel ou tel lieu, c'est en passant à proximité de, etc. À Mérignat, à l'époque actuelle, Près la cotète, Sous la Tour, Sur Vintabran constituent encore autant de localisations précisées par l'usage des prépositions à l'intention de l'interlocuteur pour lui permettre de mieux situer le lieu. La distinction à faire entre lieux de même nom s'opère grâce aux ressources variées de la langue, qu'il s'agisse de ne pas confondre Sous Côte avec La Côte en lui joignant un article, ou d'adjoindre un autre déterminant (adjectif qualificatif, complément de nom) pour différencier par exemple la Corria, de la pente Coriat.

Cela permet de mieux situer, voire de faire une distinction entre deux lieux semblables. Telles sont les motivations premières de la désignation : localiser et désigner pour survivre.

La question est alors : quand il y a localisation, qu'est-ce qui motive le choix de telle ou telle désignation? Dans la langue du locuteur, c'est le choix du mot qui va rendre compte de la réalité du lieu telle qu'elle est pensée au moment de la désignation, parmi divers possibles. Ce qui suppose de la part du locuteur une certaine perception de la réalité, ce qui suppose un accord, au moins tacite, des interlocuteurs sur la réalité ainsi désignée et l'aspect retenu, et aussi un accord sur le mot choisi. Or qu'estce-que la perception de la réalité reconnue ? Elle dépend de beaucoup d'éléments. Quant au mot retenu, il désigne la substance même du lieu, c'est le substantif, le nom. Ce peut être un mot du langage courant : le mont qui désigne un relief dont la forme est, contrairement au crêt, arrondie; la combe qui désigne un relief en creux entouré de parties hautes. Ce peut être un mot employé métaphoriquement : la Tête, qui désigne un relief dont la forme évoque une tête, ou Le Col, le Golet de Vesson.

Quand la désignation est descriptive de la réalité, le choix se porte souvent sur des termes qui désignent le relief : je viens de La Combe; un cours d'eau : j'ai traversé le Veyron; un couvert végétal : je suis passé par la Chataigneraie. Toutefois, il faut éviter les approximations qui causent les errements; il convient donc souvent d'apporter des précisions. Il est donc nécessaire de faire appel à des qualifications : Le Mont-Blanc; le MontRose. Ces distinctions touchent à des qualités morphologiques retenues : forme, taille, couleur..., ou à des aspects favorables ou défavorables, liés à des expériences concrètes - un terrain peu favorable au déplacement, ou riche en cueillette, ou que l'on suppose béni des dieux ou sous l'action de puissances maléfiques. Par exemple, à Mérignat, le choix de la qualité productive d'une terre pour désigner un lieu est bien illustré par Bénétrou: 
voilà une terre «née sous une bonne étoile» (<BENE+ASTRUCUS) - dont l'antonyme français serait «malotru».

Avec le passage à une société sédentaire et le développement de l'agriculture, d'autres types de désignation vont apparaître : ceux qui sont liés à l'habitat et son emplacement : Condate; au confluent, à son extension, à ses habitants : Paris; à la fonction de la construction, immobile pour être choisie : le Pont, le Moulin; au propriétaire : Le Mas de Boson, La Bosonnière; à l'utilisation agricole : La Chenevière; à sa réussite ou non : en Matefaim (ici non sans ambiguïté aujourd'hui, puisque le souvenir primitif de cette dénomination est perdu : est-ce un pâturage nourrissant, ou - a contrario - une couche de terre arable mince comme une crêpe, même épaisse ?) ; à la présence de signes religieux : La Terre de la Croix, Saint-Jean-le-Vieux; au souvenir d'un fait marquant : Le Grand Dua («Le grand deuil», en écho à la mort suspecte à cet endroit d'un garde forestier vers 1830, jugée comme l'assassinat par un rival, condamné puis envoyé au bagne à Toulon). L'espace habituel de déplacement qui quadrille l'espace devient à son tour point de repère : Le Haut du Goudron, car le processus de création est continu, comme le montre ce dernier toponyme; il est évident que l'apparition de la mécanisation a entraîné la disparition des chariots attelés pour les travaux agricoles, et l'usage de véhicules motorisés génère des changements : $d$ 'anciens chemins ruraux sont maintenant rendus carrossables au moins en partie; on a donc vu naître à Mérignat dans les années 1990 Le haut du goudron, toponyme qui désigne le bout du chemin où il faut laisser sa voiture pour continuer à pied.

Cette création récente s'inscrit dans une succession historique liée à l'occupation des lieux. L'étude menée sur les toponymes de la commune permet aussi d'esquisser une chronologie d'apparition des toponymes actuellement conservés, par recoupement de leurs caractères linguistiques (langue de l'étymon, forme dialectale, contexte d'emploi) avec des éléments topographiques ou historiques : on a pu ainsi repérer les «strates d'usage» où ces toponymes encore en usage ont été employés, comme il apparaît sur le graphique ci-dessous.

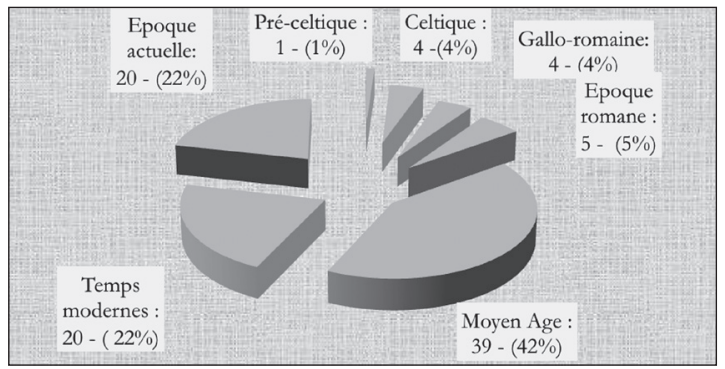

Figure 1. - Strates probables d'usage des toponymes de Mérignat. 


\section{Appropriation, transmission, conservation et remotivation d'une désignation existante}

Si la description permet de mieux comprendre où est le lieu dont on parle et qui va permettre d'agir, c'est un événement lié à ce lieu, réussite de la chasse par exemple, qui va permettre mémorisation et emploi récurrent. Ainsi, il y a fixation du terme générique qui le désigne et appropriation individuelle et collective. À chaque fois qu'il y a dénomination d'un lieu, c'est bien l'utilité pour les usagers du lieu qui constitue la motivation première, à l'échelle de la propriété, de la section cadastrale ou de la commune pour ses habitants, et le critère de sa conservation. La carte de Mérignat ci-dessous illustre le phénomène (les lieux-dits inscrits au cadastre y figurent en clair, et les toponymes d'usage oral marqués d'un point étoilé). L'observation de la répartition fait apparaître une densité plus grande autour du village et le long des chemins, alors que les confins extérieurs, plus escarpés et en bois et taillis moins exploités quotidiennement, ne reçoivent que peu de noms.

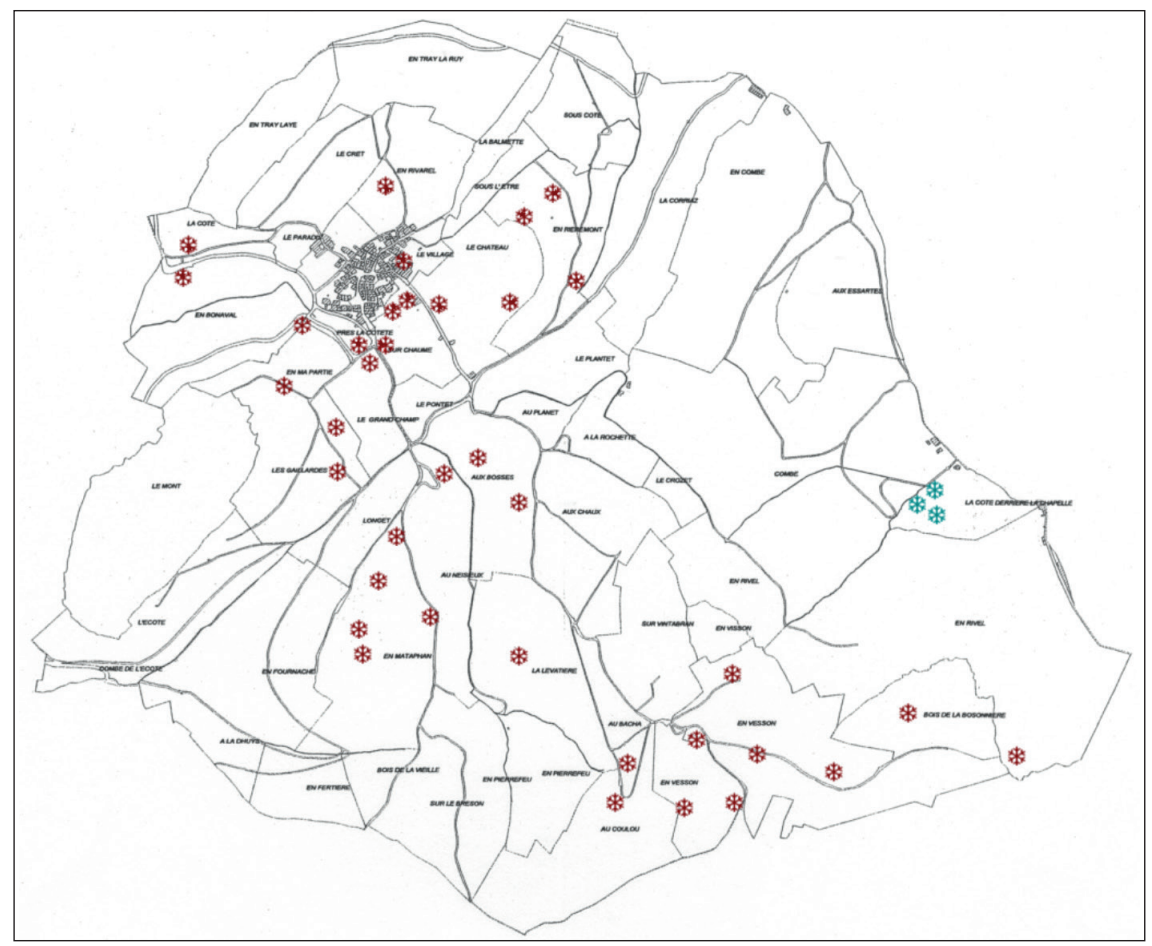

Carte de Mérignat. 
C'est la condition de la transmission collatérale et chronologique. Progressivement le nom de lieu perd de sa motivation descriptive première. Par répétition et mémorisation, il devient un nom propre. On peut dire que le nom propre de lieu constitue le niveau zéro du nom propre. Toute théorie sur le nom propre doit ainsi se référer au nom de lieu et à la motivation de son utilisation. Comme l'écrit Bosredon (2012), «la signalétique constitue bien cet espace intermédiaire de la nomination entre noms propres et noms communs» (p. 25). Repérant trois propriétés remarquables, il conclut : «dénominations hybrides, elles composent en effet deux opérations» (ibid.) que nous résumons sous les termes de dénotation et connotation.

La conservation d'une désignation peut s'expliquer par l'arbitraire qu'on sait être le propre des langues, arbitraire renforcé par le système symbolique dont se sert le groupe social qui utilise cette désignation. Mais la dénomination étant un fait de langue, ce fait constitue en soi une limite, une fragilité évidente dans la désignation que l'on voudrait stable de l'espace. En effet toute langue évolue phonétiquement et la dénomination perd de sa compréhension pour les générations suivantes. Il arrive en outre que la réalité originelle qui justifiait la dénomination ait disparu, et que le toponyme survive. À Mérignat, Le Pontet qui enjambait le ruisseau n'existe plus depuis que l'adduction d'eau du village a capté la source, vers 1860, mais le nom subsiste pour le lieu où il existait. De même, Le Biolet, maintenant en culture de vigne, pré, jardin, a perdu depuis longtemps le bois de bouleaux qui justifiait son nom. Mais le relief et l'hydrographie sont globalement stables et varient peu; ils organisent l'espace. Il peut donc arriver que dans un même ensemble sociétal il y ait nécessité de remotiver une désignation. Nombreux sont les cas de réexplication. La mémoire individuelle et collective conserve d'autant plus les noms qu'ils sont motivés par la réalité ou une image mentale, d'où la nécessité de la remotivation. Le lieu-dit cadastré En ma partie, transcription maladroite de «En Maparti» (forme francoprovençale très probable pour maupertuis «mauvais passage») se justifiait par la topographie : un verrou rocheux laissant une place étroite pour le ruisseau et un chemin, débouchant sur un ravin abrupt; la création d'un chemin départemental en 1898 l'a fait disparaître au profit d'un pont, et le souvenir s'en est perdu. Les propriétaires des terres en surplomb ont transformé le toponyme et ont baptisé l'endroit Sur mon Parti, vague réminiscence des sonorités pour une signification oubliée.

Cette remotivation peut être interne au groupe, social et linguistique, qui l'utilise, d'où des tentatives d'explication, voire de modification par les utilisateurs : Le bois de la Faye («du hêtre») devient le bois de la Fée. Mais cela peut dépendre des détenteurs du pouvoir de cartographier ou de cadastrer l'espace, lesquels vont imposer une nouvelle désignation. Le 
Fort des (de les) Cluses devient, par ignorance du francoprovençal chez les géomètres, le Fort l'Écluse.

Les événements de l'Histoire font même faire disparaitre une langue chez les peuples vaincus ou assimilés et entrainent aussi une remotivation des désignations qui leur sont empruntées. Le vainqueur dépend du vaincu pour s'assurer la maîtrise de l'espace. C'est ce que montrent les toponymes qui sont constitués de deux termes ayant un sens proche appartenant à deux langues différentes : Chalamont (du celtique *CALA «mont» et latin MONTEM «mont»); à Mérignat, Vintabran - toponyme présent dans le Midi - ressort d'un phénomène analogue, avec deux racines pré-indoeuropéennes, *VINT et *BREN, qui expriment toutes deux l'idée de «hauteur», «sommet» : la perte de sens de *VINT a entraîné probablement l'adjonction de *BREN et crée cette tautologie lorsque la dénomination primitive cesse de faire sens.

\section{Méthodologie}

Un rapide parcours dans les périodes identifiées, le Paléolithique et le Néolithique, les périodes historiques qui se sont succédé et que confirmerait une étude des sociétés récemment disparues ou en cours de disparition, permet de faire apparaître non seulement les enjeux théoriques liés à la philologie et aux sciences du langage, mais aussi liés aux sciences de l'homme sans exception (histoire, psychologie, sociologie et anthropologie) et aux sciences exactes (géologie, botanique, biologie animale). Sont concernés aussi les enjeux méthodologiques de la collecte des noms de lieux et du traitement des données recueillies.

La complexité de la situation actuelle des noms de lieux sur un territoire donné amène donc le collecteur et l'analyste des données recueillies à prendre en compte beaucoup d'éléments. Pour que l'analyste, s'il est différent du collecteur, puisse se livrer à une véritable exégèse du nom de lieu pour faire apparaitre ses significations, il faut que soit pris en compte le fait que ce mot est lié à une mémoire. Cette mémoire doit être recueillie et transcrite. À Mérignat, le toponyme d'usage oral Au Lavatroua n'est plus guère usité et beaucoup ignorent sa signification. L'informateur sollicité pour l'enquête menée a clairement exprimé le sens français de ce mot composé patois - «Lave-pressoir» -, souvenir d'une vigne d'altitude disparue depuis un siècle, où la vendange tardive donnait un jus acide, pressé en dernier, et qui «rinçait» le pressoir.

Il convient que l'analyste, aussi exigeant soit-il sur l'interprétation des microtoponymes, ne paralyse pas une collecte, devenue aujourd'hui une nécessité, en introduisant dans ses conclusions une telle imprécision ou 
un tel doute sur ses résultats qu'il conduise au désarroi des collecteurs, voire au scepticisme dont on sait qu'une des conséquences est l'abandon de l'action.

Après de telles considérations, il apparaît qu'étudier les toponymes relève bien d'une démarche scientifique touchant aux sciences et non seulement à une linguistique réduite à la pratique philologique. À partir de là un plan d'action touchant à la collecte des noms de lieux de la région Rhône-Alpes peut être mis en œuvre.

\section{Démarche adoptée dans l'enquête. Exemple : Mérignat}

Il s'agit de collecter des microtoponymes, des lieux-dits, les noms donnés à un lieu à petit échelle (une commune), que ces noms existent par écrit (cadastre, et autres documents), ou seulement par oral (dans l'usage et la mémoire des habitants). Plusieurs étapes de travail sont nécessaires pour permettre leur étude à l'échelle d'une commune.

\section{Collecter}

Si la collecte des microtoponymes écrits est nécessaire, elle peut être menée sans urgence puisque les documents sont quasi pérennes; celle des microtoponymes «oraux » s'impose comme une priorité, puisque les témoins vieillissent et disparaissent...

\section{Microtoponymes écrits}

Les cadastres constituent la base incontournable (cadastres napoléoniens du début du XIX ${ }^{\mathrm{e}}$ siècle, et cadastres actuels, consultables en Mairie et aux Archives départementales). Les situations sont très variables selon les communes : celles où ont été opérés des remembrements importants peuvent enregistrer de fortes variations avec appauvrissement du nombre des toponymes au cadastre actuel.

Les documents d'élaboration des cadastres offrent des renseignements précieux : les «états de section» enregistrent par parcelle les propriétaires, l'occupation du sol, les noms (parfois absents sur les plans cadastraux, ou avec des variantes...). À Mérignat, on enregistre peu de variations de 1826 à aujourd'hui (moins de 5\%).

Les autres documents (terriers, censiers, testaments, minutes notariales...), lorsqu'ils existent et peuvent être exploités, sont des sources précieuses, tant pour enrichir la liste des toponymes d'une commune que pour observer les variations graphiques : les documents les plus anciens donnent le témoignage le plus intéressant puisqu'il se rapproche de la 
racine qui a servi à former le toponyme, et donc du sens originel (au fil des années et des siècles, les déformations ont souvent fait perdre la signification primitive).

\section{Microtoponymes oraux}

À Mérignat, les microtoponymes oraux ont été recueillis auprès de plusieurs témoins, anciens du village (il est essentiel que les témoins aient une très bonne connaissance des lieux, de leur histoire telle qu'ils l'ont reçue).

Ce travail a été fait en plusieurs séances, en Mairie autour du cadastre napoléonien et du cadastre actuel, ainsi qu'à domicile. Au cours du travail d'enregistrement, ont été présents le souci de faciliter l'expression des témoins (toute information peut être utile, même si elle paraît sans intérêt a priori) et celui de ne pas orienter le témoignage (notamment sur les significations proposées par les témoins).

Pour chaque toponyme ont été enregistrés : prononciation, variantes, emploi en contexte (je vais à..., je viens de...), rapprochements avec d'autres lieux-dits, particularités (topographie, géologie, végétation...), traditions liées, sens et étymologie proposés. Ces rubriques sont celles de la grille d'enquête de la base de données TOPORA - Toponymes en Rhône-Alpes.

À Mérignat, cette collecte a été très fructueuse : à côté d'une cinquantaine de toponymes écrits, une cinquantaine d'autres, oraux, sont ainsi remontés à la surface et sauvés d'un oubli irrémédiable. La liste des toponymes a ainsi doublé. Et la recherche paraît aboutie.

Néanmoins, il faut être conscient que cette liste n'a rien de définitif et reste nécessairement ouverte : d'autres toponymes écrits ou oraux peuvent être retrouvés, et nous proposons une liste «En l'état actuel de la documentation » à cette date...

\section{Rechercher la base étymologique de chaque toponyme}

- Pour ce travail, il convient de bien définir l'entrée : le nom clef. En effet plusieurs cas de figure se présentent : nom simple (Le Muret) ou locution nominale (Nom + Complément : Combe de l'Ecotè Adjectif + Nom : Le Grand champ, Bonnecombe - Préposition + Nom : En tray laye - Verbe + Nom : Au Lavatroua ...). Chaque fois, même si le sens général est donné par l'intégralité du toponyme, son étude part de la racine nominale.

- Chaque fois qu'elle existe, on part des significations proposées par les témoins : pour les valider (ex. : Au bacha $=\mathrm{Bac}$ ), ou pour rétablir une étymologie plus vraisemblable que l'étymologie populaire 
(ex. : La Terre de l'herse, non pas «la terre où l'on passe la herse», mais «la terre défrichée par brûlis»).

- La recherche passe bien sûr par l'exploitation des ressources documentaires (dictionnaires, ouvrages et revues linguistiques... à l'Institut Pierre Gardette, de l'Université catholique de Lyon) : la confrontation des formes attestées permet de dégager le sens, toujours en relation avec les informations topographiques données par l'enquête de collecte.

- Cependant la prudence est de rigueur dans la validation : on obtient des certitudes (ex. : La Dhuys = captage, conduite d'eau), on enregistre le sens avec des probabilités (ex. : La Provenchère = montagne creuse), on reste avec des énigmes (ex. : Soussisrou).

\section{III.1. Élaborer une fiche}

Ce travail aboutit à l'élaboration pour chaque toponyme d'une fiche toponymique (sorte d'«état civil» du nom : localisation, particularités, sens, origine...) : elle est enregistrée dans la grille «Enquêteur» de la base de données TOPORA, installée sur le site web de l'UCLy, et consultable sur ce site (avec la totalité des informations, pour les enquêteurs et chercheurs, et une partie des informations pour le grand public).

\section{III.2. Élaborer une notice}

En vue d'une diffusion plus large, et par des moyens divers selon les situations dans les communes, l'essentiel des informations relatives à un toponyme est synthétisé dans une notice toponymique, organisée en cinq articles : Attestations du toponyme - Coordonnées dans la commune Description du lieu - Étymologie et signification retenues - Précisions complémentaires.

Les notices sont également enregistrées dans la base de données TOPORA, mais ne seront accessibles à tous que deux ans au minimum après leur édition, pour respecter les droits de diffusion des publications qui peuvent être faites.

\section{IV. Établir le corpus toponymique de la commune}

Le regroupement des toponymes d'une commune aboutit à une liste alphabétique par nom (suivi entre parenthèses de ses déterminants : préposition, article, adjectif, compléments...). Bien entendu, cette liste reste toujours provisoire et «ouverte», les ajouts ultérieurs pouvant être intégrés à la base TOPORA. 
L'intérêt d'établir cette liste est double :

- à l'échelle de la commune, elle permet - par tris successifs et rapprochements - une étude de la commune dans le temps et dans l'espace, et une meilleure connaissance de celle-ci;

- à l'échelle plus vaste de la région, elle facilite l'étude des toponymes en Rhône-Alpes (aires linguistiques francoprovençale et occitane) : la base de données consultable sera d'autant plus riche que les listes communales y seront nombreuses.

\section{Analyser le corpus}

Cette analyse peut être menée selon deux axes :

\section{Chronologie}

L'étude révèle l'histoire du peuplement de la commune :

- par la chronologie des étymons qui renvoient à une langue parlée par une population donnée à une époque donnée (ex. à Mérignat : Vintabran, d'origine néolithique). Mais l'emploi d'un mot en un lieu quelconque est, la plupart du temps, bien postérieur à sa «création» ailleurs;

- par la recherche des «Strates d'usage» (ex. : pré-celtique, celtique, gallo-romaine...) dans la commune : à quel moment tel toponyme y a-t-il été employé ? Une proposition de stratification et la méthode employée pour l'établir sont présentées dans l'ouvrage Mérignat. Étude des microtoponymes, coédité par Patrimoine des Pays de l'Ain - où il est disponible - et l'Institut Pierre Gardette.

\section{Typologie}

L'étude dégage la configuration topographique et humaine de la commune.

Le regroupement des toponymes, par type (géonyme, praxonyme) et par thème (hydronyme, oronyme, agronyme, etc.), met en valeur les caractères fondamentaux de la commune. Là aussi, une méthode est proposée dans l'ouvrage Mérignat. Étude des microtoponymes.

Cette double étude donne ainsi matière à expliciter les rapports des habitants à leur environnement, les interactions qui ont façonné le paysage et l'organisation sociale en ce lieu. 


\section{Publier}

Plusieurs moyens existent de diffuser les découvertes de ces travaux. Bien loin de s'exclure, ils se complètent les uns les autres :

- TOPORA, base de données «Toponymes de Rhône-Alpes» est le fruit de la collaboration de Patrimoine des Pays de l'Ain et de l'Institut Pierre Gardette de l'UCLy. Enrichie par les collectes au fil des travaux menés dans les communes, elle est consultable par le grand public et les chercheurs. C'est l'outil le plus complet, pour conserver et rendre accessible l'intégralité des résultats de recherche des microtoponymes de la Région.

- Conférences, promenades, expositions, présentations... Les moyens de restituer aux habitants ce que leurs anciens connaissent sont variés et peuvent être mis en œuvre dans les communes selon les possibilités et les compétences présentes. À Mérignat, deux réunions de présentations et une «promenade toponymique» ont rassemblé un public fourni et intéressé...

- Publication «papier» : avec des formats variés, il en existe déjà dans certaines communes. Nécessairement moins complète que la base TOPORA, l'édition papier poursuit des objectifs de diffusion différents et s'adresse à un public potentiel plus limité. Pour le département de l'Ain, invitation est faite, à tous ceux qui sont actuellement dans la phase de recherche, à publier leur travail dans la collection «Inventaire des noms de lieux de l'Ain» (coédition PPA-IPG).

\section{BIBLIOGRAPHIE SOMMAIRE}

Bessat Hubert et Germi Claudette, 1993, Lieux en mémoire de l'alpe. Toponymie des alpages en Savoie et Vallée d'Aoste, Grenoble, ELLUG.

BosRedon Bernard, 2012, «Entre dénomination et catégorisation : la signalétique », Langue française, Larousse, $\mathrm{n}^{\circ} 174$, p. 11-25.

CLERgET Joël (dir.), 1990, Le nom et la nomination, source, sens et pouvoirs, Toulouse, Erès.

Gerfaud Jean-Pierre et Poncet Noël, 2012, Mérignat. Étude des microtoponymes, Bourg-en-Bresse, PPA/IPG.

NÈGre Ernest, 1990-1991, Toponymie générale de la France, Genève, Droz.

Nommer l'espace, 1997, Le Monde alpin et rhodanien, ${ }^{\text {os }}$ 2-4, Grenoble, Centre alpin et rhodanien d'ethnologie.

Philipon Édouard, 1911, Dictionnaire topographique du département de l'Ain, Paris, Imprimerie nationale. 


\section{Annexes}

\section{Grille de collecte}

\begin{tabular}{|c|l|l|}
\hline & \multicolumn{1}{|c|}{ Intitulé du champ } & $\begin{array}{c}\text { Renseignements collectés } \\
\text { pour un toponyme }\end{array}$ \\
\hline 1 & No $^{\circ}$ Toponyme & \\
\hline 2 & No $^{\circ}$ INSEE de la commune & \\
\hline 3 & Nom de la commune & \\
\hline 4 & Nom du département & \\
\hline 5 & Nom du hameau & \\
\hline 6 & Toponyme (au xxl siècle) & \\
\hline 7 & Variante graphique & \\
\hline 8 & Prononciation locale & \\
\hline 9 & Emploi du toponyme en contexte («je vais à -, en -») & \\
\hline 10 & Section cadastrale & \\
\hline 11 & Lieu-dit cadastral & \\
\hline 12 & Numéro de parcelle(s) & \\
\hline 13 & Toponyme (cadastre napoléonien 1) & \\
\hline 14 & Toponyme (cadastre napoléonien 2) & \\
\hline 15 & Attestation 1 dans la commune (forme) & \\
\hline 16 & Attestation 1 dans la commune (type) & \\
\hline 17 & Attestation 1 dans la commune (date) & \\
\hline 18 & Attestation 2 dans la commune (forme) & \\
\hline 19 & Attestation 2 dans la commune (type) & \\
\hline 20 & Attestation 2 dans la commune (date) & \\
\hline 21 & Attestation 3 dans la commune (forme) & \\
\hline 22 & Attestation 3 dans la commune (type) & \\
\hline 23 & Attestation 3 dans la commune (date) & \\
\hline 24 & Autres attestations (en et/ou hors commune) & \\
\hline 25 & Déclivité & \\
\hline 26 & Nature du sol (géologie, pédologie...) & \\
\hline 27 & Couverture végétale au xIX siècle & \\
\hline 28 & Couverture végétale au xxl siècle & \\
\hline 29 & Bâti (type, utilisation) & \\
\hline 30 & Étymologie proposée & \\
\hline 31 & Tradition orale liée & \\
\hline 32 & Tradition orale liée (suite) & \\
\hline 33 & Tradition orale liée (suite) & \\
\hline 34 & Tradition orale liée (suite) & \\
\hline 35 & Renvoi à un autre toponyme & \\
\hline 36 & Nom de l'enquêteur & \\
\hline 37 & Date de l'enquête & \\
\hline 38 & Informateur(s) & \\
\hline 39 & Champ libre & \\
\hline & & \\
\hline
\end{tabular}




\section{Grille de recherche}

\begin{tabular}{|l|l|l|}
\hline & \multicolumn{1}{|c|}{ Intitulé du champ } & $\begin{array}{c}\text { Renseignements collectés } \\
\text { pour un toponyme }\end{array}$ \\
\hline 40 & Dictionnaire topographique & \\
\hline 41 & G.P.F.P. & \\
\hline 42 & G.P.S.R. & \\
\hline 43 & Noms de lieux (Vincent...) & \\
\hline 44 & TGF (Nègre) & \\
\hline 45 & DENF (Morlet) & \\
\hline 46 & Autres ouvrages & \\
\hline 47 & FEW & \\
\hline 48 & Étymon(s) retenu(s) & \\
\hline 49 & Langue de l'étymon retenu & \\
\hline 50 & Sens du toponyme & \\
\hline 51 & Forme dialectale & \\
\hline 52 & Type & \\
\hline 53 & Thème principal & \\
\hline 54 & Thème secondaire & \\
\hline 55 & Déterminant(s) & \\
\hline 56 & Prépositions(s) & \\
\hline 57 & Strate d'usage probable en ce lieu & \\
\hline
\end{tabular}

\section{Grille de rédaction}

\begin{tabular}{|l|l|l|}
\hline \multicolumn{1}{|c|}{ Intitulé du champ } & $\begin{array}{c}\text { Renseignements collectés } \\
\text { pour un toponyme }\end{array}$ \\
\hline 58 & Notice rédigée : A- attestations & \\
\hline 59 & Notice rédigée : C- coordonnées & \\
\hline 60 & Notice rédigée : D- description & \\
\hline 61 & Notice rédigée : E- étymologie & \\
\hline 62 & Notice rédigée : P- précisions & \\
\hline 63 & Rédacteur & \\
\hline
\end{tabular}

